\title{
Parameter selection in particle swarm optimisation: a survey
}

\begin{abstract}
Nowadays, particle swarm optimisation (PSO) is one of the most commonly used optimisation techniques. However, PSO parameters significantly affect its computational behaviour. That is, while it exposes desirable computational behaviour with some settings, it does not behave so by some other settings, so the way for setting them is of high importance. This paper explains and discusses thoroughly about various existent strategies for setting PSO parameters, provides some hints for its parameter setting and presents some proposals for future research on this area. There exists no other paper in literature that discusses the setting process for all PSO parameters. Using the guidelines of this paper can be strongly useful for researchers in optimisation-related fields.
\end{abstract}

Keyword: Particle swarm optimisation; Artificial intelligence; Optimisation; Parameter selection 